\title{
The function and morphology of Meibomian glands in patients with thyroid eye disease: a preliminary study
}

\author{
Chia-Yu Wang ${ }^{1 \dagger}$, Ren-Wen Ho ${ }^{2,3+}$, Po-Chiung Fang ${ }^{2}$, Hun-Ju Yu², Chun-Chih Chien ${ }^{4}$, Chang-Chun Hsiao ${ }^{5}$ \\ and Ming-Tse Kuo ${ }^{2^{*}}$ (D)
}

\begin{abstract}
Background: To investigate function and morphology of the meibomian gland (MG) in patients with thyroid eye disease (TED).

Methods: In this prospective case series study, patients with unilateral or bilateral TED were consecutively enrolled. The diagnosis of TED was based on the typical orbital findings and/or radiographic evidence. The disease activity of TED was classified according to the clinical activity score (CAS). Degrees of lagophthalmos and exophthalmos, blinking rates, and results of the Schirmer test 1 were also recorded. All patients completed the SPEED questionnaire and underwent MG assessment, including lipid layer thickness (LLT), MG dropout (MGd), and MG expression.

Results: In total 31 eyes from 17 patients with unilateral or bilateral TED were included. Patients were divided into inactive TED (CAS 0-1; 20 eyes from 11 patients) and active TED (CAS 2-3, 11 eyes from 6 patients) groups. MGd was significantly more severe in the active TED than the inactive TED group [Median (Inter-quartile region): $3.0(2.0-3.0)$ vs. $2.0(1.0-2.0)$ degree, $P=0.04]$. However, patients with active TED had thicker LLT than those with inactive TED (90.0 [80.0-100.0] vs. $65.0[47.8-82.5] \mathrm{nm}, P=0.02)$, and LLT was positively correlated with lagophthalmos $(r=0.37, P=0.04)$.

Conclusions: Patients with active TED had more severe MGd, but thicker LLT. Active TED may cause periglandular inflammation of MGs, leading to MGd, but compensatory secretion from residual MGs and lagophthalmos-induced forceful blinking might temporarily release more lipids over the tear film.
\end{abstract}

Keywords: Lipid layer thickness, Meibomian gland dysfunction, Thyroid eye diseases

\section{Background}

Thyroid eye disease (TED), also known as Graves' ophthalmopathy and thyroid-associated orbitopathy, is an ocular manifestation of a systemic autoimmune disorder. The orbit presents the same antigens as the thyroid gland, such as the thyroid-stimulating hormone receptor, thyrotropin receptor, and insulin-like receptor [1]. Consequently, for patients with immune-related thyroid dysfunction, the circulating autoantibodies may also

\footnotetext{
* Correspondence: mingtse@cgmh.org.tw

${ }^{\dagger}$ Equal contributors

2Department of Ophthalmology, Kaohsiung Chang Gung Memorial Hospital and Chang Gung University College of Medicine, Kaohsiung, Taiwan Full list of author information is available at the end of the article
}

attack the orbit by triggering a cytokine cascade and causing orbital fibroblast proliferation, adipose tissue expansion, and glycosaminoglycan secretion [2]. Finally, patients may develop lid edema, chemosis, lid retraction, exophthalmos, lagophthalmos, restrictive myopathy, and compressive optic neuropathy, and may complain of diplopia and decreased vision [3].

Dry eye disease (DED) is very common in patients with TED: the prevalence rate of DED in TED is up to $65.2 \%[4,5]$. Coulter et al. reported that $97 \%$ of patients with TED in a cohort study had dry eye symptoms [6]. Some underlying mechanisms have been proposed. First, lid retraction, exophthalmos, and lagophthalmos may cause ocular surface changes and blinking abnormalities

(c) The Author(s). 2018 Open Access This article is distributed under the terms of the Creative Commons Attribution 4.0 International License (http://creativecommons.org/licenses/by/4.0/), which permits unrestricted use, distribution, and reproduction in any medium, provided you give appropriate credit to the original author(s) and the source, provide a link to the Creative Commons license, and indicate if changes were made. The Creative Commons Public Domain Dedication waiver (http://creativecommons.org/publicdomain/zero/1.0/) applies to the data made available in this article, unless otherwise stated. 
$[2,7,8]$, which increase the evaporation of tears and lead to DED in patients with TED [2, 9]. Second, lacrimal acinar cells physiologically express thyroid-stimulating hormone receptors [10]; thus, the antigen-antibody reaction of TED may impair the lacrimal gland and subsequently result in a decreased volume of reflex tearing $[10,11]$. Third, TED may disturb the secretion of aqueous tears and make the tear film unstable, leading to shorter tear film breakup time and increased tear film osmolality [7, 10-12].

However, increasing evidence indicates that meibomian gland (MG) dysfunction is a major risk factor of DED $[13,14]$. The International Dry Eye Work Shop have classified DED into aqueous tear deficiency (ATD) and evaporative dry eye (EDE) $[15,16]$, and recognized MG dysfunction as the primary cause of EDE [17]. Similar to patients with MG dysfunction, patients with TED usually also have dry eye symptoms. However, previous studies only focused on the ATD in patients with TED but neglected the MG dysfunction in these patients. MGs, which are special sebaceous glands in the eyelids, secrete lipids to stabilize the tear film, decrease the surface tension, and prevent the evaporation of aqueous tears [18]. MGs are arranged in parallel palisades throughout the tarsus plates of the eyelids, and the blinking motion serves as a pumping force that releases the meibomian lipids, which are formed by meibocytes within the acini, onto the lid margin [19, 20]. TED may cause eyelid inflammation, disturb the blinking motion, and gradually change the ocular surface environment as it progresses. Therefore, we hypothesized that TED may influence the performance of MGs, similar to many systemic inflammatory diseases, such as Sjögren syndrome, psoriasis, and rosacea [21-24], causing MG dysfunction. The aim of the present preliminarily study was to investigate the performance of MGs in patients with TED.

\section{Methods \\ Subjects}

This study was a prospective case series study, which formed part of an investigation of ocular adnexal microorganisms. All procedures involving human subjects adhered to the Declaration of Helsinki. Institutional Review Board (IRB)/Ethics Committee approval was obtained from the Committee of Medical Ethics and Human Experiments of Chang Gung Memorial Hospital $(\mathrm{CGMH})$, Taiwan. Informed consent was obtained from each subject in the CGMH.

Patients with hyperthyroidism and unilateral or bilateral TED were included. All participants were asked not to instill topical eye drops for $4 \mathrm{~h}$ and ointment for $12 \mathrm{~h}$ before examination. Subjects with other systemic diseases (e.g., hypertension, diabetes mellitus, connective tissue diseases, etc.), had undergone previous eyelid and ocular surgeries, or had super-active TED (clinical activity score $[\mathrm{CAS}] \geq 4$ ) $[25]$ were excluded from this study. Seventeen age-sex-laterality-matched participants, who visited our clinics and met the same criteria, except for the absence of TED, were enrolled as a control group.

\section{Diagnosis of thyroid eye disease}

Diagnosis of TED was made on the basis of 2 of the following 3 criteria [26]. First, at least 1 immune-related thyroid dysfunction (Grave's hyperthyroidism, Hashimoto thyroiditis, circulating thyroid antibody) was present. Second, the imaging study revealed fusiform enlargement of at least 1 of the ocular muscles. Third, patients had at least 1 of the following typical orbital signs: upper eyelid retraction, exophthalmos, typical restrictive strabismus, fluctuating lid edema, or chemosis/caruncular edema [27].

Among the above ocular signs, the extent of exophthalmos was measured using Hertel's exophthalmometer, which measures the distance of the corneal apex from the level of the lateral orbital rim [28]. The amount of incomplete or defective closure of eyelids (lagophthalmos) was measured [29]. The duration of thyroid disease, from the onset of hyperthyroidism to receiving the ocular examination of this study, was recorded.

\section{Classification of patients with thyroid eye disease by clinical activity score}

The disease activity in patients with TED was scored according to the CAS clinical criteria proposed by Mourits et al. [25] This score contains 7 items including pain at rest, painful eye movement, red eyelid, red conjunctiva, swelling of the eyelid, chemosis, and swollen caruncle. Each item scores 1 point, so that each eye of a TED patient has a CAS score that can range from 0 to 7 . For the purposes of our study, we defined inactive TED as CAS $0-1$ and active TED as CAS 2-3 (Fig. 1). Superactive TED patients (CAS score $\geq 4$ ) were excluded from this study, because the patients had unstable ocular conditions and received pulse corticosteroid therapy.

\section{Measurement of blinking rates and eyelid patterns}

The blinking motion of the eyelids was recorded and analyzed using a LipiView ${ }^{\circledR}$ II Ocular Surface Interferometer (TearScience, Inc., Morrisville, NC, USA) [30]. Blinks without complete closure were automatically distinguished from those with complete closure by this instrument. The partial blinking rate of each eye, the ratio of incomplete blinks to total blinks within $20 \mathrm{~s}$, was recorded for analysis.

\section{Assessment of amount of tears}

Schirmer test 1 (ST-1) was used to evaluate the tear amount of each subject [31]. Without providing topical 

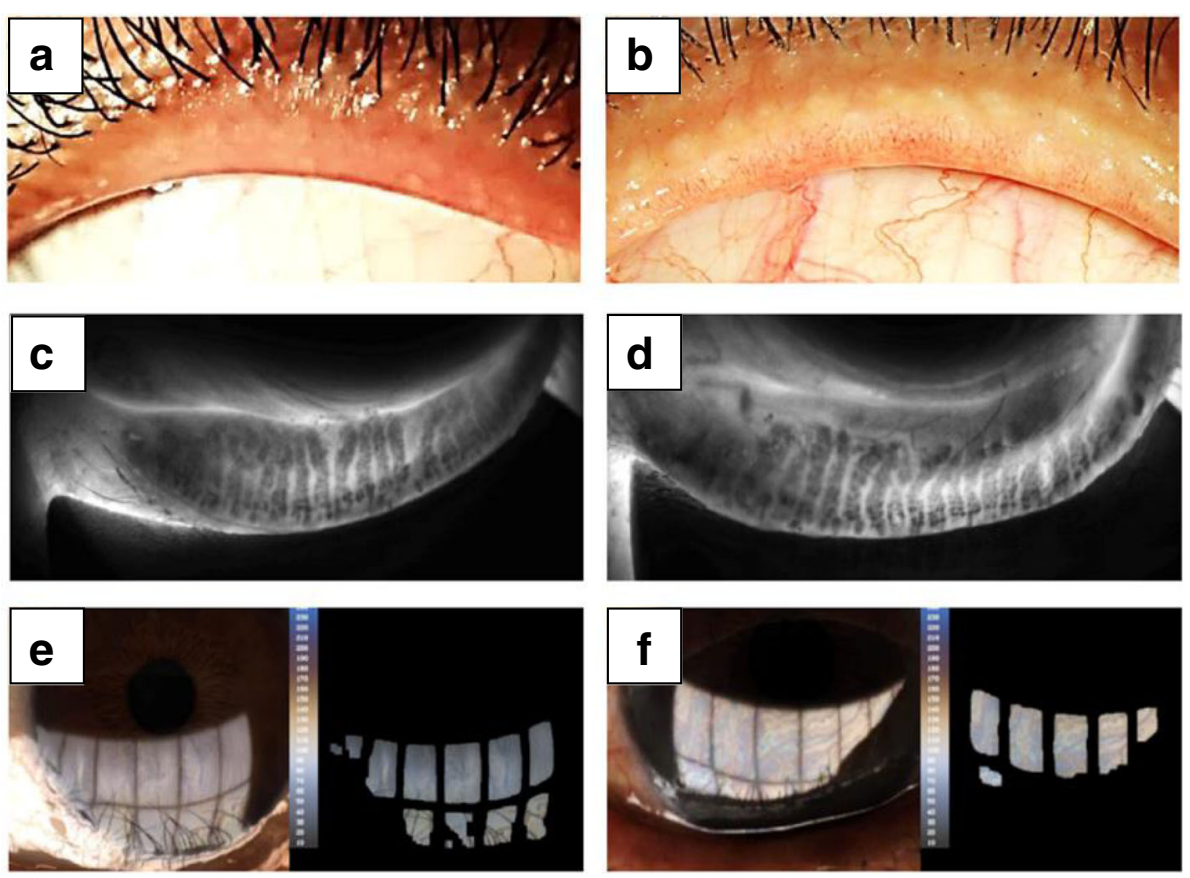

Fig. 1 Representative photos and LipidView ${ }^{\otimes} \|$ images of inactive $(\mathbf{a}, \mathbf{c}, \mathbf{e})$ and active $(\mathbf{b}, \mathbf{d}, \mathbf{f})$ thyroid eye diseases. $\mathbf{a}, \mathbf{b}$ External eye photos of upper lid margins (OS). a) A 37-year-old female with inactive thyroid eye disease (CAS 1). Upper lid margin showed no pouting or capping of meibomian gland orifices. Only mild telangiectasia was observed. b) A 49-year-old female with active thyroid eye disease (CAS 3). Upper lid margin showed pouting and plugging of meibomian gland orifices. Telangiectasia was also present. $\mathbf{c}$, $\mathbf{d}$ Infrared images of meibomian gland of the left lower lid. c) Grade 1 meibomian gland dropout (0-25\%). d) Grade 2 meibomian gland dropout (25-50\%). e, f Image of lipid layer (OS). e) Average lipid layer thickness: $79 \mathrm{~nm}$ f) Average lipid layer thickness: $100^{+} \mathrm{nm}$

anesthesia, the Schirmer strip was suspended on the inferior eyelid between the inner two-thirds and the outer onethird for $5 \mathrm{~min}$. The length of the wetting part on the test strip was then recorded for each eye.

Standard patient evaluation of eye dryness questionnaire The Standard Patient Evaluation of Eye Dryness (SPEED) questionnaire [32], designed for assessing the symptoms of dry eye, was used for these subjects. According to the frequency and severity of dry eye symptoms, the SPEED score ranges from 0 to 28, with asymptomatic patients scoring 0 and the most symptomatic patients scoring 28 .

\section{Eyelid signs representing meibomian gland dysfunction}

Each eye of TED patients was carefully assessed for representative signs of MG dysfunction, including plugging of MG orifices, lid margin irregularities, thickening, vascular engorgement, and mucocutaneous junction shift (Fig. 1a, b) [33, 34]. Eyes with any of the above signs were designated as MG dysfunction sign-positive.

\section{Grading of meibomian gland dropout}

The structure of the MGs of each eye was assessed using meibography, a near-infrared (NIR) illumination captured using the LipiView ${ }^{\circ}$ II Ocular Surface Interferometer
(TearScience, Inc., Morrisville, NC). For standardization and minimization of invasiveness, only MGs in the lower eyelid were examined. The severity of MG dropout (MGd) was classified into degree 0 to degree 4 according to the meiboscale proposed by Pult et al. [35], in which the score increases by 1 degree for every $25 \%$ of MG loss (Fig. 1c, d).

Determination of meibum quality and meibomian gland expressibility

An MG evaluator (TearScience, Inc.), which can provide a stable pressure mimicking the pressure of the orbicularis oculi muscle on MGs during normal blinking was used to press the central lower eyelid for about 10-15 s for each patient [36]. Then, the meibum quality of each gland was scored from 0 to 3 points (0: clear liquid secretion; 1: cloudy liquid secretion; 2 : cloudy particulate fluid; 3: inspissated, similar to toothpaste) [37]. MG expressibility (MGE) for each patient was recorded by counting the expressible glands (glands with scores of 0 , 1 , or 2) from the 8 glands of the central lower lid. Scores of 0,1 , and 2 indicates that $\geq 5$ glands, $3-4$ glands, and 1 -2 glands are expressible, respectively; a score of 3 indicates that no gland is expressible. MGs yielding liquid secretion (MGYLS) were also recorded by counting the glands showing liquid secretion (glands with scores 0 or 1). Moreover, a total meibum quality score (TMQS) was 
defined by summing the scores from the 8 glands in the central lower lid.

\section{Lipid layer thickness of tear film}

The lipid layer thickness (LLT) of the tear film of each eye was measured and recorded using a LipiView $^{\circ}$ II Ocular Surface Interferometer (TearScience, Inc.) (Fig. 1e, f) [38]. An average interferometric color unit (ICU) $(1 \mathrm{ICU}=1 \mathrm{~nm})$ was used for quantification of LLT. Because the exact value of LLT cannot be estimated precisely or shown by this instrument if the value exceeds $100 \mathrm{~nm}$, we set the LLT to $100 \mathrm{~nm}$ in cases where LLT $\geq 100 \mathrm{~nm}$.

\section{Order of testing procedures}

First, after each participant had completed the informed consent form, we observed the participant's ocular surface by slit lamp, measuring the extent of exophthalmos and lagophthalmos, and recorded the SPEED questionnaire. Second, the blinking rate and LLT were simultaneously obtained by a LipiView ${ }^{\circ}$ II Ocular Surface Interferometer, after which the structures of the MGs of the bilateral lower eyelids were sequentially assessed using the same instrument. Third, the Oxford staining score for each eye was recorded. Fourth, after each participant rested for about $30 \mathrm{~min}$, ST-1 was carried out for 5 min. Finally, meibum quality and MG expressibility were determined using the MG evaluator.

\section{Statistical analysis}

Statistical analyses were performed in SPSS version 20.0 for Windows (IBM Corp, Armonk, NY). Wilcoxon signed-rank tests and Fisher's exact tests were used to compare the TED patients and non-TED participants. Wilcoxon's rank-sum test was used to compare the differences of parameters between the inactive and active TED groups. Spearman's rank correlation was used to examine the correlation between parameters. $P$-values $<.05$ were considered as statistically significant. Using a free power calculator (G* power; http://www.gpower. hhu.de), the sample size of at least 15 eyes was estimated based on the comparison of ST-1 and tear film break-up time between Graves' disease patients and normal subjects according to Bruscolini A et al. [39] under the power of 0.95 .

\section{Results}

\section{Participants}

A total of 17 patients with TED (31 eyes) were collected consecutively from the Oculoplasty Clinic of CGMH between October 2015 and June 2016. Thirty-one age-sex -laterality-matched non-TED eyes were consecutively enrolled for comparison. The clinical profiles of these subjects are summarized in Table 1 . When comparing the
TED with the non-TED group, the indices of TED complications, including degrees of exophthalmos and lagophthalmos, were significantly different between the groups. However, there was no statistically significant difference in partial blinking rate, tear volume, or SPEED score. Moreover, among the indices of MG performance, only MG dysfunction signs and MGYLS showed statistically significantly differences between TED and non-TED eyes.

\section{Comparison between active and inactive TED from the indices of TED complications}

When we compared the active and inactive TED eyes, the degrees of exophthalmos and lagophthalmos were significantly higher in active TED eyes $(P=.01$ and $P<.001$, respectively), while the partial blinking rate and ST-1 were not statistically significantly different $(P=.83$ and $P=.20$, respectively). Moreover, there was no significant difference in the SPEED score between the active and inactive TED patients $(P=.80)$ (Table 2$)$.

\section{MG performance in active and inactive TED eyes}

Among the parameters of MG performance, MGd and LLT were statistically significantly different between active and inactive TED eyes. MGd was significantly more severe in active TED eyes than in inactive TED eyes $(P=.03)$. However, in conflict with the inference from the MGd result, active TED eyes had significantly thicker LLT than did those with inactive TED $(P=.02)$. There were high proportions of TED patients with MG dysfunction signs. All active TED eyes and up to $80 \%$ of inactive TED eyes had signs of MG dysfunction, but there was no statistically significant difference between the 2 groups $(P=.27)$. Moreover, the 2 groups did not show significant differences in MG expression, including MGE $(P=.15)$, MGYLS $(P=.36)$, and TMQS $(P=.48)$ (Table 2 and Fig. 2).

\section{Correlation between MG performance and TED complications}

To investigate the impact of TED activity on MG performance, a correlation analysis between the parameters of MG performance and the indices of TED complications was performed. There was no significant association between MGd and TED complications, including exophthalmos, lagophthalmos, and partial blinking rate (Fig. 3). However, a significantly positive correlation was found between LLT and lagophthalmos $(r=0.37, P=.04)$, but no other parameters of TED complications correlated with LLT.

\section{Discussion}

The impact of MG dysfunction, the leading cause of DED, on patients with TED, has remained unclear. Interestingly, 
Table 1 The clinical characteristics of the study subjects

\begin{tabular}{llll}
\hline & $\begin{array}{l}\text { TED group } \\
\text { (31 eyes of 17 patients) }\end{array}$ & $\begin{array}{l}\text { Non-TED group } \\
\text { (31 eyes of 17 controls) }\end{array}$ & $\begin{array}{c}P \text { value } \\
\text { Age (yr) }\end{array}$ \\
CAS & $44.7 \pm 11.0$ & $44.7 \pm 11.2$ & - \\
Disease duration (months) & $1.6 \pm 0.7$ & - & - \\
Exophthalmos (mm) & $47.3 \pm 63.5$ & $16.5 \pm 1.1$ & $<0.001^{*}$ \\
Lagophthalmos (mm) & $20.4 \pm 4.3$ & $0.0 \pm 0.0$ & $0.015^{*}$ \\
Partial blinking rate (\%) & $0.9 \pm 1.9$ & $55.1 \pm 34.1$ & 0.702 \\
ST-1 (mm) & $58.4 \pm 34.7$ & $13.0 \pm 10.2$ & 0.885 \\
SPEED (score) & $12.6 \pm 9.0$ & $6.8 \pm 4.7$ & 0.509 \\
MG dysfunction signs ${ }^{\ddagger}$ (eyes) & $7.4 \pm 4.1$ & 1 & $<0.001^{*}$ \\
MGd (score) & 27 & $2.2 \pm 0.9$ & 0.555 \\
MGE (score) & $2.1 \pm 0.9$ & $0.8 \pm 0.9$ & 0.379 \\
MGYS (score) & $0.6 \pm 1.1$ & $3.0 \pm 2.5$ & $0.014^{*}$ \\
TMQS (score) & $4.7 \pm 3.1$ & $8.5 \pm 4.9$ & 0.412 \\
LLT (nm) & $10.1 \pm 8.0$ & $72.0 \pm 21.9$ & 0.654 \\
\hline
\end{tabular}

tFisher's exact test was used to test the between-group differences in MG dysfunction signs, while the Wilcoxon signed-rank test was used to test other parameters

(*) Statistically significant $(P<0.05)$

‡MG dysfunction signs, signs of meibomian gland (MG) dysfunction, including irregular lid margin, vascular engorgement, plugged meibomian gland orifices, and displacement of mucocutaneous junction

CAS Clinical activity score, ST-1 Schirmer test 1, SPEED Scores of SPEED questionnaire, MGd MG dropout, MGE MG expressibility, MGYLS MG yielding liquid secretion, TMQS Total meibum quality score, LLT Lipid layer thickness

Table 2 Comparison between active and inactive thyroid eye diseases

\begin{tabular}{llll}
\hline & $\begin{array}{l}\text { Active TED } \\
(11 \text { eyes of }\end{array}$ & $\begin{array}{l}\text { Inactive TED } \\
\text { (20 eyes of } \\
\text { 6 patients) }\end{array}$ & 11 patients) \\
\hline Age (yr) & $45.2 \pm 16.1$ & $44.4 \pm 7.3$ & 0.679 \\
Sex (female: male) & $7: 4$ & $11: 9$ & 0.718 \\
Disease duration (months) & $80.8 \pm 96.8$ & $28.9 \pm 20.5$ & 0.264 \\
Exophthalmos (mm) & $23.1 \pm 4.3$ & $18.5 \pm 3.3$ & $0.005^{*}$ \\
Lagophthalmos (mm) & $2.13 \pm 2.67$ & $0.18 \pm 0.59$ & $<0.001^{*}$ \\
Partial blinking rate (\%) & $58.2 \pm 29.9$ & $58.5 \pm 37.8$ & 0.834 \\
ST-1 (mm) & $9.2 \pm 5.6$ & $14.5 \pm 10.0$ & 0.199 \\
SPEED (score) & $7.1 \pm 4.6$ & $7.6 \pm 3.9$ & $16(80 \%)$ \\
MG dysfunction signs ${ }^{*}$ (no. of eyes; \%) & $11(100 \%)$ & $1.8 \pm 0.7$ & 0.803 \\
MGd (score) & $2.5 \pm 0.9$ & $0.9 \pm 1.2$ & 0.269 \\
MGE (score) & $0.2 \pm 0.4$ & $4.3 \pm 3.1$ & $0.03^{*}$ \\
MGYLS (score) & $5.4 \pm 2.9$ & $11.2 \pm 9.0$ & 0.148 \\
TMQS (score) & $8.2 \pm 5.5$ & $67.4 \pm 21.0$ & 0.362 \\
LLT (nm) & $86.3 \pm 18.0$ & 0.482 & $0.024^{*}$ \\
\hline
\end{tabular}

${ }_{t, \pm}$ Patients with thyroid eye diseases (TED) were classified by the clinical activity score (CAS) as having active TED (CAS 2-3) or inactive TED (CAS 0-1); ${ }^{5}$ Fisher's exact test was used to test the between-group differences in sex and MG dysfunction signs, while Wilcoxon's rank-sum test was used to test other parameters. (") Statistically significant $(P<0.05)$

"MG dysfunction signs, signs of meibomian gland (MG) dysfunction, including irregular lid margin, vascular engorgement, plugged meibomian gland orifices, and displacement of mucocutaneous junction 

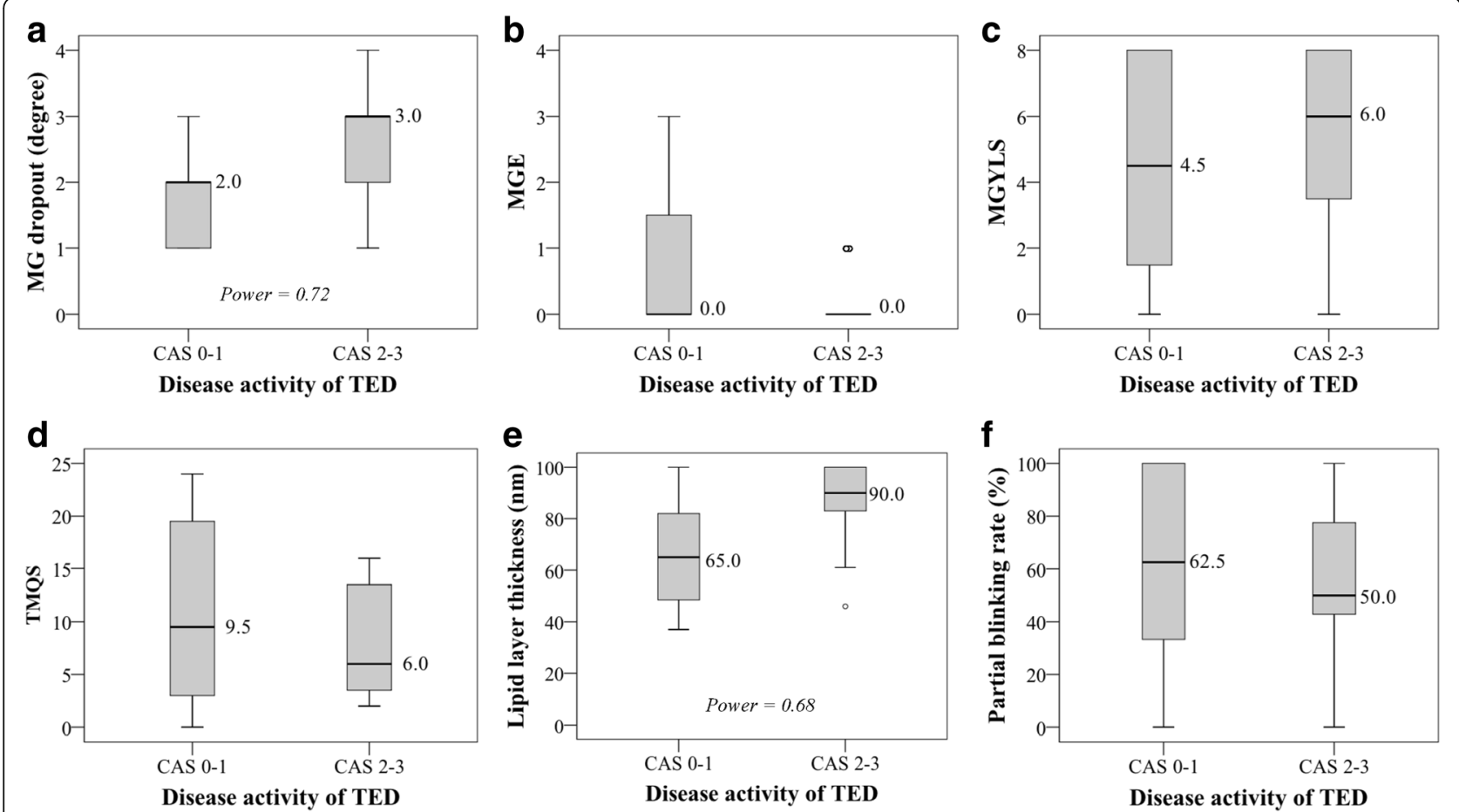

Fig. 2 Comparison between active and inactive thyroid eye diseases (TED) for meibomian gland dropout (MGd), meibomian gland expression, including meibomian gland expressibility (MGE), meibomian gland yielding liquid secretion (MGYLS), and total meibum quality score (TMQS), as well as lipid layer thickness (LLT), and partial blinking rate, by boxplot diagrams. The dot in Fig. 2b shows the 2 outliers of MGE in CAS 2-3. Box, 25th to 75th percentile; bold line in the box, median; bars, minimum and maximum values; dot, outliers

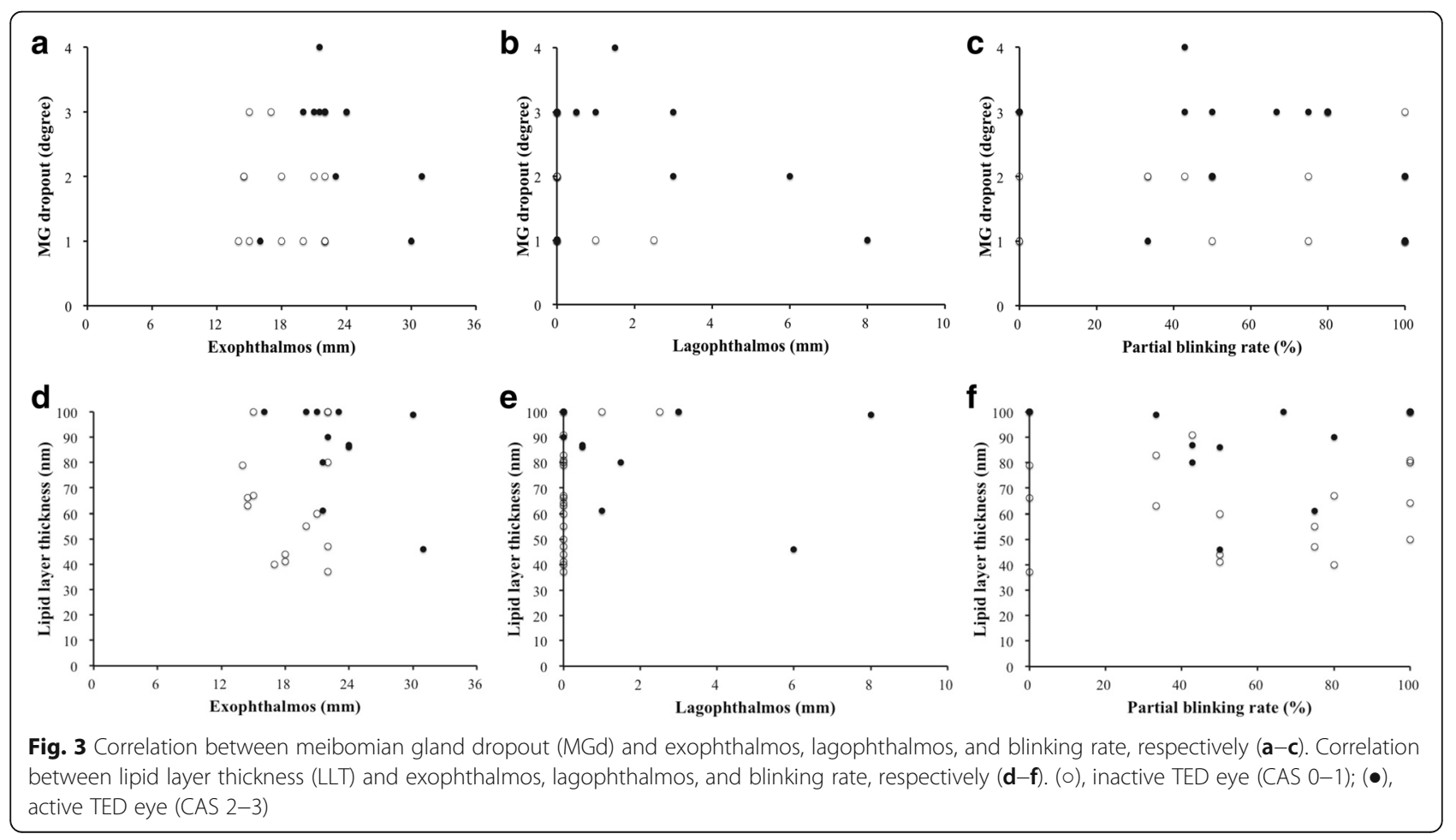


27 eyes (87.1\%) had signs of MG dysfunction, but the mean age of these TED patients was only 44.7 years (Table 1 ). We also found that active TED eyes (CAS 2-3) had a higher MGd, but thicker LLT, than inactive TED eyes (CAS 0-1) (Table 2 and Fig. 2). Although active TED eyes had more severe exophthalmos and lagophthalmos, only lagophthalmos was associated with a thicker LLT (Fig. 3). The finding of a thicker LLT, but higher MGd, in active TED suggests not only compensatory activity from the residual MGs, but also that lagophthalmos-mediated forceful blinking is involved. This could be a potential mechanism for decreasing ocular surface injury from the more severe lagophthalmos in active TED.

Active ocular inflammation may cause typical ocular surface changes in TED in patients with thyroid disease [2]. We hypothesized that active TED would further impede the performance of the MGs in these patients. Although the CAS score proposed by Mourits to reflect the inflammation status of TED ranged from 0 to 7 [25], only patients with a CAS of less than 4 were included in this study. All patients with CAS exceeding 3 received pulse-corticosteroid treatment, and they were excluded from this study to prevent a possible bias due to this treatment. A flair-up of TED may result in greater extraocular muscle enlargement, and this may be reflected in the greater exophthalmos and lagophthalmos of the active TED eyes in this study (Table 2).

In this study, the mean MGd of TED patients was 2.1 (Table 1), representing about $25-50 \%$ loss of MGs. Active TED eyes had significantly greater loss of MGs than inactive TED eyes (Table 2). However, loss of MGs was not associated with the target indices of TED complications (Figs. 3a, b, and c). Several studies have shown a strong association between MGd and inflammatory ocular surface diseases. Mathers et al. reported that patients with chronic blepharitis and giant papillary conjunctivitis demonstrated a greater loss of MGs [40, 41]. Shimazaki et al. reported that Sjögren syndrome was associated with MG dysfunction [22]. Knop et al. pointed out that inflammatory mediators could spread and lead to glandular dropout and potentially to acinar atrophy by way of the conjunctiva, through the tarsus and toward the MGs [42]. Thus, TED-associated ocular surface inflammation might cause periglandular inflammation, and subsequent loss of MGs.

A recent study proposed that a thinner LLT may predict a higher risk of MG dysfunction [43]. Eom et al. also found that greater loss of MGs is correlated with a thinner LLT [44]. It is reasonable that a stasis of lipid inside the MGs may increase pressure in the MGs, causing the ducts to dilate, and finally resulting in acinar atrophy [19]. However, in our study, the active TED eyes showed a greater loss of MGs, but thicker LLT, than the inactive TED eyes (Table 2 and Fig. 2). Additionally, we found a positive correlation between lagophthalmos and LLT (Fig. 3), and active TED eyes had greater lagophthalmos than inactive TED eyes (Table 2). Kim et al. reported that some MGs may be obstructive and atrophic, while other MGs may secrete lipids at normal or enhanced levels to compensate for MG dysfunction, whereby normal LLT is maintained [45]. Korb et al. found that forceful blinking could increase the LLT [46]. It is possible that active TED patients had more severe MGd, but thicker LLT, not only as a compensatory effect, but also as a stimulatory effect. The compensatory effect may be induced by MGd, based to some degree on a physiological response from residual MGs, but not on the over-production of lipids. However, patients with more severe lagophthalmos may show more severe punctate erosion on the ocular surface. Additionally, MG disease may increase corneal sensitivity [47]. Therefore, active TED patients with more severe lagophthalmos may have pathological lipid hypersecretion due to forceful blinks. Patients with TED might blink more forcefully, unconsciously, due to greater lagophthalmos. Although the active TED eyes demonstrated more severe MGd, the mixed compensatory and stimulatory effect may cause temporarily thicker LLT than that seen in inactive TED eyes.

In addition, the function of MGs may be maintained at a certain level, because the loss of MGs is partial (on average $25-50 \%$ ) even in active TED eyes. Active TED eyes had greater exophthalmos than inactive TED eyes (Table 2 and Fig. 2). Exophthalmos could stretch the eyelid, inducing even higher lid tension and making it easier for the lipids in the MGs to be squeezed out. However, there was no significant correlation between exophthalmos and LLT (Fig. 3).

All but 1 of the patients with TED suffered from dry eye symptoms, as identified on the SPEED questionnaire. Despite the lack of statistically significant difference, lower aqueous tear secretion (ST-1) was found in patients with active TED $(9.2 \pm 5.6)$ than in patients with inactive TED and in the normal control group (14.5 \pm 10.0 and $13.0 \pm 10.2$, respectively). These findings were compatible with those of Eckstein et al. [10], who concluded that ATD may be caused by diminished lacrimal gland function in active TED. The expression of inactive TED may be the same as proposed by Arita et al. [48], who pointed out that increased tear fluid is produced as a temporary compensatory response to loss of MGs.

There were some limitations in this preliminary study. The non-TED control group may be suitable for comparison with the TED group in our clinical practice, but this control group cannot truly represent a normal population. Some participants in the control group also had dry eye symptoms and inadequate MG performance. Both eyes of bilateral TED patients were pooled with the 
3 eyes of the 3 unilateral TED patients. We had found similar results in the analyses of right eyes or left eyes. Although a trend for thicker LLT in active TED was noted, the wide range of standard deviation resulted in a non-significant difference. Thus, we classified both eyes of the same patient into the same CAS group, which might have caused a bias. However, all patients with active TED (CAS 2-3) in our study had ocular signs of similar severity, only 2 patients with inactive TED (CAS $0-1$ ) had single eye involvement. One eye in the active TED group was excluded due to previous eyelid surgery. Furthermore, most patients routinely used eye ointment for lubrication at night. The usage of topical ointment should be more strictly limited to avoid its influence on LLT. The ingredients of an eye ointment might affect the tear film composition, yet all patients had ceased ointment application at least $12 \mathrm{~h}$ before examination. Moreover, we set LLT as $100 \mathrm{~nm}$ for the 5 eyes with LLT $>100 \mathrm{~nm}$, which may have caused underestimation of the average value of LLT. Because the LipiView ${ }^{\circ}$ II Ocular Surface Interferometer did not have a sensor to identify the blinking force, we cannot clearly prove the association between LLT and forceful blinks. A further study adopting simultaneous electromyography of the eyelid should be considered to verify this causality. Finally, the small sample size implies that our results should be interpreted with some caution. Subgroup analysis revealed that inactive TED eyes were not significantly different from non-TED eyes in terms of MG performance and LLT, but a trend for greater MGd and thicker LLT was observed between active TED eyes and non-TED eyes. Thus, a small proportion of active TED patients in our subjects might be the reason for the lack of statistically significant differences in many parameters between the TED group and the non-TED group. The performance of MGs in TED patients should be verified in a future study with a larger sample.

\section{Conclusions}

In conclusion, the results of this study indicated that patients with active TED had more severe MGd, but thicker LLT. Active TED may cause periglandular inflammation of MGs, leading to MGd, although lagophthalmos might induce a compensatory effect, involving increased lipid secretion from the residual MGs in an attempt to stabilize the tear film.

\footnotetext{
Abbreviations

ATD: Aqueous tear deficiency; CAS: Clinical activity score; DED: Dry eye disease; EDE: Evaporative dry eye; LLT: Lipid layer thickness; MG: Meibomian gland; MGd: Meibomian gland dropout; MGE: Meibomian gland expressibility; MGYLS: Meibomian gland yielding liquid secretion; SPEED: Standard patient evaluation of eye dryness; ST-1: Schirmer test 1; TED: Thyroid eye disease; TMQS: Total meibum quality score
}

\section{Acknowledgments}

The authors would like to thank all colleagues who contributed to this study. We are grateful to Prof. Sheng-Nan Lu, Prof. Hsueh-Wen Chang, Chih-Yun Lin, Shin-Yi Chien, and the Biostatistics and Bioinformatics Center, Kaohsiung Chang Gung Memorial Hospital for assistance with statistical analyses.

\section{Funding}

This work was supported by Chang Gung Research Proposal (CMRPG8C0762), and the Ministry of Science and Technology (Grant No. 104-2314-B-182A-101$\mathrm{MY} 3$ ). The sponsors or funding organizations had no role in the design or conduct of this research.

\section{Availability of data and materials}

The datasets used and analyzed during the current study are available from the corresponding author on reasonable request.

\section{Authors' contributions}

CYW analyzed and interpreted the data, and was a major contributor in writing the manuscript. RWH designed and conducted the study, and a contributor in writing and reviewing the manuscript. CYW and RWH contributed to this manuscript equally. PCF designed the study and reviewed the manuscript. HJY reviewed the manuscript. CCC analyzed and interpreted the data. CCH reviewed the manuscript. MTK helped RWH to collect the patients' data and reviewed the manuscript. All authors read and approved the final manuscript.

\section{Ethics approval and consent to participate}

Institutional Review Board (IRB)/Ethics Committee approval was obtained from the Committee of Medical Ethics and Human Experiments of Chang Gung Memorial Hospital (CGMH), Taiwan. Informed consent was obtained from each subject in the CGMH.

\section{Consent for publication}

Not applicable.

\section{Competing interests}

The authors declare that they have no competing interests.

\section{Publisher's Note}

Springer Nature remains neutral with regard to jurisdictional claims in published maps and institutional affiliations.

\section{Author details}

${ }^{1}$ Department of Ophthalmology, Taipei Tzu Chi Hospital, Buddhist Tzu Chi Medical Foundation, Taipei, Taiwan. ${ }^{2}$ Department of Ophthalmology, Kaohsiung Chang Gung Memorial Hospital and Chang Gung University College of Medicine, Kaohsiung, Taiwan. ${ }^{3}$ Graduate Institute of Clinical Medicine, College of Medicine, Kaohsiung Medical University, Kaohsiung, Taiwan. ${ }^{4}$ Department of Laboratory Medicine, Kaohsiung Chang Gung Memorial Hospital and Chang Gung University College of Medicine, Kaohsiung, Taiwan. ${ }^{5}$ Graduate Institute of Clinical Medical Sciences, Chang Gung University, Taoyuan City, Taiwan.

Received: 9 August 2017 Accepted: 3 April 2018

Published online: 12 April 2018

References

1. Bartalena L, Tanda ML. Clinical practice. Graves' ophthalmopathy. N Engl J Med. 2009;360(10):994-1001.

2. Sokol JA, Foulks GN, Haider A, Nunery WR. Ocular surface effects of thyroid disease. Ocul Surf. 2010;8(1):29-39.

3. Bartley GB, Fatourechi V, Kadrmas EF, Jacobsen SJ, Ilstrup DM, Garrity JA, Gorman CA. Clinical features of Graves' ophthalmopathy in an incidence cohort. Am J Ophthalmol. 1996;121(3):284-90.

4. Achtsidis V, Kozanidou E, Bournas P, Tentolouris N, Theodossiadis PG. Dry eye and clinical disease of tear film, diagnosis and management. Eur Ophthalmic Rev. 2014;8(1):17-22.

5. Ismailova DS, Fedorov AA, Grusha YO. Ocular surface changes in thyroid eye disease. Orbit. 2013;32(2):87-90.

6. Coulter I, Frewin S, Krassas GE, Perros P. Psychological implications of Graves' orbitopathy. Eur J Endocrinol. 2007;157(2):127-31. 
7. Gilbard JP, Farris RL. Ocular surface drying and tear film osmolarity in thyroid eye disease. Acta Ophthalmol. 1983;61(1):108-16.

8. Cruz AA, Ribeiro SF, Garcia DM, Akaishi PM, Pinto CT. Graves upper eyelid retraction. Surv Ophthalmol. 2013:58(1):63-76.

9. Schiffman RM, Christianson MD, Jacobsen G, Hirsch JD, Reis BL. Reliability and validity of the ocular surface disease index. Arch Ophthalmol. 2000; 118(5):615-21

10. Eckstein AK, Finkenrath A, Heiligenhaus A, Renzing-Kohler K, Esser J, Kruger C, Quadbeck B, Steuhl KP, Gieseler RK. Dry eye syndrome in thyroidassociated ophthalmopathy: lacrimal expression of TSH receptor suggests involvement of TSHR-specific autoantibodies. Acta Ophthalmol Scand. 2004; 82(3 Pt 1):291-7.

11. Chang TC, Huang KM, Chang TJ, Lin SL. Correlation of orbital computed tomography and antibodies in patients with hyperthyroid Graves' disease. Clin Endocrinol. 1990;32(5):551-8.

12. Gupta A, Sadeghi PB, Akpek EK. Occult thyroid eye disease in patients presenting with dry eye symptoms. Am J Ophthalmol. 2009;147(5):919-23.

13. Viso E, Gude F, Rodriguez-Ares MT. The association of meibomian gland dysfunction and other common ocular diseases with dry eye: a populationbased study in Spain. Cornea. 2011;30(1):1-6.

14. Tong L, Chaurasia SS, Mehta JS, Beuerman RW. Screening for meibomian gland disease: its relation to dry eye subtypes and symptoms in a tertiary referral clinic in Singapore. Invest Ophthalmol Vis Sci. 2010;51(7):3449-54.

15. Lemp MA. Report of the National eye Institute/industry workshop on clinical trials in dry eyes. CLAO J. 1995:21(4):221-32.

16. Lemp MA: The 1998 Castroviejo lecture. New strategies in the treatment of dry-eye states. Cornea 1999, 18(6):625-632.

17. Bron AJ, Tiffany JM. The contribution of meibomian disease to dry eye. Ocul Surf. 2004;2(2):149-65.

18. Bron AJ, Tiffany JM, Gouveia SM, Yokoi N, Voon LW. Functional aspects of the tear film lipid layer. Exp Eye Res. 2004;78(3):347-60.

19. Knop E, Knop N, Millar T, Obata H, Sullivan DA. The international workshop on meibomian gland dysfunction: report of the subcommittee on anatomy, physiology, and pathophysiology of the meibomian gland. Invest Ophthalmol Vis Sci. 2011;52(4):1938-78.

20. Knop N, Knop E. Meibomian glands. Part I: anatomy, embryology and histology of the Meibomian glands. Ophthalmologe. 2009;106(10):872-83.

21. Schaumberg DA, Nichols JJ, Papas EB, Tong L, Uchino M, Nichols KK. The international workshop on meibomian gland dysfunction: report of the subcommittee on the epidemiology of, and associated risk factors for, MGD. Invest Ophthalmol Vis Sci. 2011;52(4):1994-2005.

22. Shimazaki J, Goto E, Ono M, Shimmura S, Tsubota K. Meibomian gland dysfunction in patients with Sjogren syndrome. Ophthalmology. 1998; 105(8):1485-8.

23. Horwath-Winter J, Flogel I, Ramschak-Schwarzer S, Hofer A, Kroisel PM Psoriasis and hypogonadism in chronic blepharokeratoconjunctivitis. A case report. Ophthalmologe. 2002;99(5):380-3.

24. Alvarenga LS, Mannis MJ. Ocular rosacea. Ocul Surf. 2005;3(1):41-58.

25. Mourits MP, Koornneef L, Wiersinga WM, Prummel MF, Berghout A, van der Gaag R. Clinical criteria for the assessment of disease activity in Graves' ophthalmopathy: a novel approach. Br J Ophthalmol. 1989;73(8):639-44.

26. Bartley GB, Gorman CA. Diagnostic criteria for Graves' ophthalmopathy. Am J Ophthalmol. 1995;119(6):792-5.

27. Bahn RS. Graves' Ophthalmopathy. N Engl J Med. 2010;362(8):726-38.

28. Onofrey BE, Skorin L, Holdeman NR. Ocular therapeutics handbook : a clinical manual. 3rd ed. Philadelphia: Wolters Kluwer/Lippincott Williams \& Wilkins; 2012. p. 71-72

29. Pereira MV, Gloria AL. Lagophthalmos. Semin Ophthalmol. 2010;25(3):72-8.

30. Satjawatcharaphong P, Ge S, Lin MC. Clinical outcomes associated with thermal pulsation system treatment. Optom Vis Sci. 2015;92(9):e334-41.

31. American Academy of Ophthalmology. BCSC section 8. External disease and cornea. Sutphin JE ed. San Francisco: American Academy of Ophthalmology; 2009-2010. p. 62.

32. Ngo W, Situ P, Keir N, Korb D, Blackie C, Simpson T. Psychometric properties and validation of the standard patient evaluation of eye dryness questionnaire. Cornea. 2013;32(9):1204-10.

33. Driver PJ, Lemp MA. Meibomian gland dysfunction. Surv Ophthalmol. 1996; 40(5):343-67.

34. Bron AJ, Benjamin L, Snibson GR. Meibomian gland disease. Classification and grading of lid changes. Eye (Lond). 1991;5(Pt 4):395-411.
35. Pult H, Riede-Pult B. Comparison of subjective grading and objective assessment in meibography. Cont Lens Anterior Eye. 36(1):22-7.

36. Korb DR, Blackie CA. Meibomian gland diagnostic expressibility: correlation with dry eye symptoms and gland location. Cornea. 2008;27(10):1142-7.

37. Tomlinson A, Bron AJ, Korb DR, Amano S, Paugh JR, Pearce El, Yee R, Yokoi $\mathrm{N}$, Arita R, Dogru M. The international workshop on meibomian gland dysfunction: report of the diagnosis subcommittee. Invest Ophthalmol Vis Sci. 2011;52(4):2006-49.

38. Zhao Y, Tan CL, Tong L. Intra-observer and inter-observer repeatability of ocular surface interferometer in measuring lipid layer thickness. BMC Ophthalmol. 2015;15:53.

39. Bruscolini A, Abbouda A, Locuratolo N, Restivo L, Trimboli P, Romanelli F. Dry eye syndrome in non-exophthalmic Graves' disease. Semin Ophthalmol. 2015;30(5-6):372-6.

40. Mathers WD, Shields WJ, Sachdev MS, Petroll WM, Jester JV. Meibomian gland dysfunction in chronic blepharitis. Cornea. 1991;10(4):277-85.

41. Mathers WD, Billborough M. Meibomian gland function and giant papillary conjunctivitis. Am J Ophthalmol. 1992;114(2):188-92.

42. Knop E, Knop N. Meibomian glands : part IV. Functional interactions in the pathogenesis of meibomian gland dysfunction (MGD). Ophthalmologe. 2009;106(11):980-7.

43. Finis D, Pischel N, Schrader S, Geerling G. Evaluation of lipid layer thickness measurement of the tear film as a diagnostic tool for Meibomian gland dysfunction. Cornea. 2013;32(12):1549-53.

44. Eom Y, Lee JS, Kang SY, Kim HM, Song JS. Correlation between quantitative measurements of tear film lipid layer thickness and meibomian gland loss in patients with obstructive meibomian gland dysfunction and normal controls. Am J Ophthalmol. 2013;155(6):1104-10.

45. Kim HM, Eom Y, Song JS: The relationship between morphology and function of the meibomian glands. Eye Contact Lens 2016 [PMID: 27755288; Epub ahead of print].

46. Korb DR, Baron DF, Herman JP, Finnemore VM, Exford JM, Hermosa JL, Leahy CD, Glonek T, Greiner JV. Tear film lipid layer thickness as a function of blinking. Cornea. 1994;13(4):354-9.

47. Rahman EZ, Lam PK, Chu CK, Moore Q, Pflugfelder SC. Corneal sensitivity in tear dysfunction and its correlation with clinical parameters and blink rate. Am J Ophthalmol. 2015:160(5):858-66.

48. Arita R, Morishige N, Koh S, Shirakawa R, Kawashima M, Sakimoto T, Suzuki T. Tsubota K. Increased tear fluid production as a compensatory response to meibomian gland loss: a multicenter cross-sectional study. Ophthalmology. 2015;122(5):925-33.

Ready to submit your research? Choose BMC and benefit from

- fast, convenient online submission

- thorough peer review by experienced researchers in your field

- rapid publication on acceptance

- support for research data, including large and complex data types

- gold Open Access which fosters wider collaboration and increased citations

- maximum visibility for your research: over $100 \mathrm{M}$ website views per year

At BMC, research is always in progress.

Learn more biomedcentral.com/submissions 